\title{
Review of management and treatment of peritoneal metastases from gastric cancer origin
}

\author{
Israel Manzanedo ${ }^{1,2}$, Fernando Pereira ${ }^{1}$, Ángel Serrano ${ }^{1}$, Estíbalitz Pérez-Viejo ${ }^{1}$ \\ ${ }^{1}$ Department of General and Digestive Surgery, Peritoneal Carcinomatosis Unit, Hospital of Fuenlabrada, Fuenlabrada, Madrid, Spain; ${ }^{2}$ Rey Juan \\ Carlos University (URJC), Madrid, Spain \\ Contributions: (I) Conception and design: I Manzanedo; (II) Administrative support: All authors; (III) Provision of study materials or patients: All \\ authors; (IV) Collection and assembly of data: I Manzanedo; (V) Data analysis and interpretation: I Manzanedo, F Pereira; (VI) Manuscript writing: \\ All authors; (VII) Final approval of manuscript: All authors. \\ Correspondence to: Israel Manzanedo, MD. Department of General and Digestive Surgery, Hospital of Fuenlabrada, C/Camino del Molino s/n, CP: \\ 28942. Fuenlabrada, Madrid, Spain. Email: israel.manzanedo@salud.madrid.org.
}

\begin{abstract}
Gastric cancer (GC) is the third cause of cancer-related deaths in the world, with less than $25 \%$ survivors at 5 years. These results are largely related to the high incidence of peritoneal metastases (PM) in these patients. Nowadays, the standard treatment for GC with PM is palliative systemic chemotherapy (SCT) with a survival of 6 months. From the 2000s, the combination of cytoreductive surgery (CRS) with hyperthermic intraperitoneal chemotherapy (HIPEC) has been gaining popularity for different neoplastic diseases that involve the peritoneal surface. The use of CRS and HIPEC has been studied for GC with PM, with promising results in selected patients, obtaining survival rates never seen before. Moreover, HIPEC and other intraperitoneal chemotherapy techniques have been used to prevent peritoneal recurrences in patients diagnosed on locally advanced GC without macroscopic PM (adjuvant or prophylactic HIPEC). Even, intraperitoneal chemotherapy [laparoscopic HIPEC and neoadjuvant intraperitoneal and systemic chemotherapy (NIPS)] has been used as neoadjuvant treatment to reduce peritoneal disease burden in order to improve the rate of patients in whom complete cytoreduction can be achieved. Finally, patients with high volume peritoneal disease can be treated by palliative intraperitoneal chemotherapy to control the symptoms resulting from malignant ascites, using laparoscopic HIPEC or pressurized intraperitoneal aerosol chemotherapy (PIPAC). This review aims to update the management of PM from GC origin in these different clinical scenarios, based on the literature and the experience of the authors.
\end{abstract}

Keywords: Gastric cancer (GC); peritoneal metastases (PM); hyperthermic intraperitoneal chemotherapy (HIPEC); cytoreductive surgery (CRS); early postoperative intraperitoneal chemotherapy (EPIC); pressurized intraperitoneal aerosol chemotherapy (PIPAC)

Submitted Jun 09, 2020. Accepted for publication Jul 03, 2020.

doi: 10.21037/jgo-20-232

View this article at: http://dx.doi.org/10.21037/jgo-20-232

\section{Introduction}

Gastric cancer (GC) is a very aggressive disease and is the third leading cause of cancer-related death in the world, despite of being the fifth most common cancer $(1,2)$. Only $25 \%$ of patients will survive at 5 years $(3,4)$.

These poor results are mainly related to its prevalent diagnosis in metastatic stages, especially with peritoneal disease (20-30\% of patients have peritoneal dissemination at diagnosis), and $40-60 \%$ of patients will have a peritoneal relapse as the only site of recurrence after curative gastrectomy $(3,5,6)$. Nowadays, the treatment for metastatic GC is palliative systemic chemotherapy (SCT); advances in SCT have improved the prognosis of patients with metastatic GC, reaching 8-14 months in selected patients (6-9), but these results are worse when peritoneal metastases 
(PM) are present (10-12), with a survival lower than 6 months and without 5 -year survivors $(3,13)$.

The presence of isolated positive peritoneal cytology (microscopic PM), in absence of visible nodules, is classified as M1 and has a similar prognosis to that of macroscopic PM; the international consensus guidelines recommend treating these patients with palliative SCT as the other metastatic GC patients $(4,6,11)$.

The study and knowledge of PM development since the 1990s has changed the classic concept that classified $\mathrm{PM}$ as a systemic disease, being recategorized as a locally disseminated disease (14). The multimodal treatment of $\mathrm{PM}$, including SCT, cytoreductive surgery (CRS) and hyperthermic intraperitoneal chemotherapy (HIPEC), has been gaining popularity for different neoplastic diseases that involve the peritoneal surface. Macroscopic disease is treated by CRS, which includes peritonectomy procedures, and intraperitoneal chemotherapy is used to treat microscopic residual disease (15-17). Currently, this approach is the preferred treatment for pseudomyxoma peritonei, peritoneal mesothelioma and in selected patients of PM from colorectal cancer origin $(6,18-20)$; in ovarian cancer, it is well known that intraperitoneal chemotherapy works, and there is increasing evidence that HIPEC is also effective (21). For PM from GC there is also an increasing evidence based mainly on retrospective information and there are several clinical trials underway $(6,11,12,22-26)$. The survival results are encouraging in selected cases.

This multimodal treatment is an intensive approach that must be applied just in those patients who will have a benefit. For this reason, the volume of peritoneal disease must be carefully evaluated to select potentially curable patients. The primary tumor is diagnosed by endoscopy and ultrasonographic endoscopy to determine the cTN stage (27). A thoracic-abdominopelvic computed tomography (CT) with double contrast must be carried out in all cases, and a positron emission tomography (PET) in doubtful cases. Diagnostic laparoscopy is required to assess the peritoneal extension of the disease using the Peritoneal Cancer Index (PCI) (28), and to obtain a peritoneal cytology.

Because there is still no consensus on management of patients with GC and PM, we believe this review is necessary. This review aims to update the management of PM from GC origin in the different clinical scenarios, based on the literature and the experience of the authors.

We present the following article in accordance with the Narrative reporting checklist (available at http://dx.doi. org/10.21037/jgo-20-232).

\section{Methods}

This review has been made with an exhaustive search in different online databases, (PubMed, Medline, Cinahl, Embase), since 1980 until 2020, using "gastric cancer", "carcinomatosis", "peritoneal metastases", "HIPEC", "PIPAC" and others as the key words. Also, we have used our personal database with the approval of the Ethics Committee of our Institution.

\section{Importance of the volume of peritoneal disease}

In addition to the tumor's own factors (pTN stage, tumor differentiation, perineural invasion and/or signet-ring cells) $(6,11,12,22,23,29)$, the two most important prognostic factors for patients diagnosed with GC and PM are the volume of peritoneal disease (estimated by the PCI), and the possibility to achieve a complete cytoreduction, according to the Completeness Cytoreduction Score (CCS-0) (30). As both factors (PCI and CCS) are related, PCI assessment is essential to select patients for CRS with HIPEC.

The French Registry was published in 2010 by Glehen et al. (12). Their analysis of 159 patients with PM from GC origin treated with CRS and HIPEC showed a median overall survival (OS) of 9.2 months (1-, 3- and 5-year survival of $43 \%, 18 \%$ and $13 \%$, respectively), but median OS was 15 months if CRS was complete (CCS-0). No patient with PCI higher than 19 survived at 6 months, and no patient with PCI $>12$ survived at 3 years, so they recommended that patients with $\mathrm{PCI}>12$ should not been treated with CRS and HIPEC, because the benefit is extremely low or null. Since then, patients with PCI higher than 12 are usually rejected for CRS and HIPEC.

In the last years, several studies have reported an even lower PCI limit for maximum survival benefit. When patients have a PCI lower than 7 and a complete cytoreduction (CCS-0) is achieved, there is a real possibility of cure. Chia et al. published in 2016 an analysis of 81 patients treated with complete CRS (CCS- 0 or CCS-1) and HIPEC. Patients with PCI $<7$ had a median OS of 26.4 versus 10.9 months for patients with PCI $\geq 7$ (24). Our Spanish Registry was published in 2019 with an analysis of 88 patients with PM from GC origin; patients with PCI $<7$ had a median OS of 26.1 months (OS at 1-, 3- and 5-year of $88.7 \%, 46.8 \%$ and $46.8 \%$ ) while those with PCI $\geq 7$ had a median OS of 18.9 months (OS at 1-, 3- and 5-year of 
$70.4 \%, 15.1 \%$ and $0.0 \%$ ); in the multivariate analysis, the only prognostic factor that had an independent negative influence on OS was PCI higher than 6, with HR of 2.37 (95\% CI: 1.26-4.46, P=0.007) (6). Moreover, those patients with isolated positive peritoneal cytology (without macroscopic implants) had not reached their median OS, with a long follow-up. More recently, in 2020, the German Registry has been published with 235 patients; the median OS is significantly different according to PCI: median OS of 18 months for patients with PCI 0-6, 12 months for PCI 7-15 and 5 months with PCI 16-39 (31).

\section{Pre- and post-operative SCT}

After diagnosis, patients with limited and potentially resectable peritoneal disease should be treated with a multimodal approach. In western countries (Europe and United States), pre- and post-operative chemotherapy is the standard of treatment. The MAGIC trial was published in 2006 (32), and its chemotherapy scheme, with 3 preoperative and 3 postoperative courses of ECF/ECX (epirubicin, cisplatin and fluorouracil or capecitabine), has been the standard therapy for patients without metastatic disease until recently. The publication in 2019 of FLOT4 trial has changed this standard of care, and, currently, FLOT (fluorouracil, leucovorin, oxaliplatin and docetaxel) with 4 preoperative and 4 postoperative cycles is preferred because of its superior results, in localized tumors with peritoneal disease, and even at other metastatic sites (33).

\section{Neoadjuvant intraperitoneal chemotherapy}

The use of neoadjuvant intraperitoneal chemotherapy aims to achieve a decrease of peritoneal disease burden in order to enhance the number of patients in whom complete cytoreduction can be achieved. Several approaches have been studied to administer intraperitoneal chemotherapy.

The use of preoperative laparoscopic HIPEC has been recently studied (34,35). Yonemura et al., in 2017, reported 53 patients treated with 2 cycles of laparoscopic neoadjuvant HIPEC (with a combination of docetaxel and cisplatin), evaluating the benefit of this treatment by the results of peritoneal cytology and the PCI determination. A significant PCI decrease was observed (from a median of 14.2 to a median of 11.8 at the time of second session) and the cytology changed from positive to negative in $68 \%$ of cases (34). Badgwell et al. published a phase-II trial with 19 patients in 2017. They used laparoscopic HIPEC with mitomycin $\mathrm{C}$ plus cisplatin (from 1 to 5 cycles). The morbidity was $11 \%$. The peritoneal disease (macroscopic and microscopic disease) disappeared in 7 patients after the last laparoscopic HIPEC cycle (35).

The combination of neoadjuvant intraperitoneal and systemic chemotherapy (NIPS) was developed in 2006 by Yonemura (36). In patients with PM from GC origin, a peritoneal port system was introduced into the peritoneal cavity, and a combination of intraperitoneal taxotere and carboplatin with intravenous methotrexate and 5-FU was administered (between 2 and 6 cycles); the treatment achieved a negative peritoneal cytology in $56 \%$ of patients and 38 of 61 patients showed a partial response (36). In 2012, Yonemura et al. published the results of NIPS with intraperitoneal taxotere and cisplatin combined with systemic S-1. Ninety-six patients were enrolled in the study and 82 patients were eligible for CRS. Complete cytoreduction (CCS-0) was achieved in $70 \%$ and $36.8 \%$ of patients had complete pathologic response (37). Other studies have showed similar results with different NIPS regimens (38-40).

\section{CRS and HIPEC}

A complete cytoreduction is the main requirement to prolong survival. The CRS must be carried out in referral centers with demonstrated experience in the selection and treatment of peritoneal diseases (6). The first aim of CRS is to remove all visible disease by gastrectomy, D2 lymphadenectomy and removal of all peritoneal implants, which may include peritonectomies and visceral resections. Peritonectomies procedures were described by Sugarbaker. Their knowledgeable performance is mandatory to complete these complex procedures (15). The surgeon can remove all macroscopic disease, but the residual microscopic disease is the most probable cause of future relapses. The use of HIPEC tries to eliminate this microscopical disease through high cytostatic doses acting locally, with the synergy and the effect of heat (41).

CRS with HIPEC is the treatment with the best results published for patients with PM from GC origin (Table 1). Despite these promising published results, treatment with CRS and HIPEC is not yet the standard-of-care. There are retrospective series and case-control studies that show a potential benefit with CRS and HIPEC. There is one randomized clinical trial published. In 2011, Yang et al. randomized 68 patients to CRS alone or CRS with HIPEC (mitomycin $\mathrm{C}$ and cisplatin). They reported a significant but 
Table 1 Published studies evaluating hyperthermic intraperitoneal chemotherapy (HIPEC) as treatment of peritoneal metastases from gastric cancer origin

\begin{tabular}{|c|c|c|c|c|}
\hline Author, year & Number of patients & Drug & Morbidity & Survival \\
\hline Glehen 2010, (12) & 159 (CRS + HIPEC) & $\begin{array}{l}\text { MMC, Cisplatin, } \\
\text { Oxaliplatin }\end{array}$ & $\begin{array}{c}\text { Grade } 3-4: 27.8 \% . \\
\text { Mortality: } 6.5 \%\end{array}$ & $\begin{array}{c}\text { Median OS: } 9.2 \text { months. } \\
\text { 5-year OS: } 13 \%\end{array}$ \\
\hline Yang 2011, (25) & $\begin{array}{c}34 \text { (CRS + HIPEC) vs. } \\
34 \text { (CRS) }\end{array}$ & MMC + Cisplatin & $\begin{array}{c}14.7 \% \text { vs. } 11.7 \% . \\
\text { Mortality: } 0 \%\end{array}$ & $\begin{array}{l}\text { Median OS: } 11 \text { vs. } \\
6.5 \text { months }\end{array}$ \\
\hline Manzanedo 2019, (6) & 88 (CRS + HIPEC) & $\begin{array}{l}\text { Cisplatin, Doxorubicin, } \\
\text { MMC, Oxaliplatin }\end{array}$ & $\begin{array}{c}\text { Grade III-IV D-C: } 31 \% . \\
\text { Mortality: } 3.4 \%\end{array}$ & $\begin{array}{c}\text { Median OS: } 21.2 \text { months. } \\
\text { 3-year OS: } 30.9 \%\end{array}$ \\
\hline Bonnot 2019, (29) & $\begin{array}{c}180 \text { (CRS + HIPEC) vs. } \\
97 \text { (CRS) }\end{array}$ & $\begin{array}{l}\text { MMC, Cisplatin, } \\
\text { Oxaliplatin }\end{array}$ & $\begin{array}{c}53.7 \% \text { vs. } 55.3 \% . \\
\text { Mortality: } 7.4 \% \text { vs. } 10.1 \%\end{array}$ & $\begin{array}{c}\text { Median OS: } 18.8 \text { vs. } \\
12.1 \text { months. } 5 \text {-year OS: } \\
19.9 \% \text { vs. } 6.4 \%\end{array}$ \\
\hline
\end{tabular}

CRS, cytoreductive surgery; MMC, mitomycin C; NA, not available; OS, overall survival; D-C, Dindo-Clavien Classification (44).

modest improvement in survival when HIPEC was added to CRS (OS of 6.5 months for CRS alone versus 11 months for CRS with HIPEC, with 3-year survival rates of $0 \%$ and $5.9 \%$ respectively) (25).

There are three National Registries with a high volume of patients published in western countries. The French Registry was published in 2010 by Glehen et al., with 159 patients from 15 centers; they reported a median OS of 9.2 months (1-, 3- and 5-year survival of $43 \%, 18 \%$ and $13 \%$, respectively). If CRS was complete (CCS-0), the median OS rose to 15 months. They recommended that patients with PCI higher than 12 should not been treated with CRS and HIPEC, because the survival results in these patients were the same as with SCT (12).

We published the Spanish Registry in 2019. This registry included 88 patients from 7 specialized Spanish Institutions. With a median follow-up of 32 months, the median OS reached 21.2 months. The median OS at 1 -year was $79.9 \%$ and at 3 -year $30.9 \%$. The patients with PCI 0-6 had the best results with a median OS of 26.1 months (1-, 3- and 5 -year survival of $88.7 \%, 46.8 \%$ and $46.8 \%$, respectively), compared to 18.9 months of median OS in patients with PCI $>6$ (1-, 3- and 5-year survival of $70.4 \%, 15.1 \%$ and $0.0 \%$, respectively). These differences were significant in the comparative analysis with a HR of 2.23 (95\% CI: 1.28$3.88, \mathrm{P}=0.003)$. According to these results, we propose a
PCI of 7 as the cut-off point for selecting patients with GC and PM for CRS and HIPEC (6).

The German Registry was published recently, in 2020. This study includes 235 patients from 38 centers. They describe a median OS of 13 months, but the OS increases to 18 months in the group of patients with PCI $0-6$. The best results have been reached in high volume centers (with more than 20 patients included in the study); the OS in experienced centers is significantly better than in low volume centers ( 16 versus 12 months, $\mathrm{P}=0.02$ ) (31).

In 2019, Bonnot et al. published the French propensity score study CYTO-CHIP, comparing 180 patients treated with CRS and HIPEC versus 97 patients treated only with CRS. The patients treated with HIPEC had better survival than those treated without HIPEC, with a median OS of 18.8 versus 12.1 months, without morbidity and mortality differences (29).

Our Peritoneal Carcinomatosis Unit (Hospital of Fuenlabrada) is a reference center in Spain. Since 2006, we have performed more than 700 procedures in patients with PM. Until December 2019, we have operated 60 patients with PM from GC origin, with a median PCI of 6 (range, 0-35) including 10 patients with isolated positive cytology (without macroscopic PM). We have achieved a complete cytoreduction (CCS-0 or CCS-1) in 50 patients (83.3\%). Twenty-one patients (35\%) had severe complications, 
grade III-V according to Dindo-Clavien classification (44), including 2 deaths (3.3\%). With a median follow-up of 27.2 months, the median OS is 16.9 months and 5-year OS is $23.2 \%$. In the 33 patients with PCI 0-6 the median OS is 18.6 months, significantly better than in the 27 patients with PCI $>6$ (12.4 months, $\mathrm{P}=0.016)$. The 10 patients with isolated positive cytology (PCI 0) have the best results, with a median OS not reached yet, and 5-year OS of $60 \%$, versus $28.6 \%$ and median OS of 18 months in the group of 23 patients with PCI 1-6 ( $\mathrm{P}=0.019)$. These results confirm the need for careful patient selection in reference centers, because these procedures have significant morbidity and it has been shown that the best oncological results are achieved in experienced centers.

Currently, there are several Asian trials recruiting patients. Also, there is one ongoing German phase III trial (GASTRIPEC, NCT02158988) and one Dutch clinical trial (PERISCOPE II, NCT03348150) evaluating the role of HIPEC for PM of GC origin. In a few years, we will have more information from these trials that are currently underway.

There remain many questions about HIPEC as yet without an answer. The administration technique, the drug, the carrier solution and the time of administration have not been compared in any clinical trial. The different studies published show the benefit of HIPEC without differences in all these issues $(6,11,12,22,31)$. The most common drugs used in PM from GC origin are cisplatin (with or without doxorubicin) and mitomycin $\mathrm{C}$, alone or in combination; the dose for cisplatin varies between 50 to $200 \mathrm{mg} / \mathrm{m}^{2}$ during 60 or 90 minutes, and for mitomycin the dose most commonly used is $30-40 \mathrm{mg}$ during 60-120 minutes $(3,4,6,23,25,45)$. Oxaliplatin is also used with a dose of $460 \mathrm{mg} / \mathrm{m}^{2}$ during 30 minutes $(3,6,12,46)$.

\section{Early postoperative intraperitoneal chemotherapy (EPIC)}

EPIC is administered and recovered through an inflow catheter and drains placed during the CRS, from the first postoperative day to the $4^{\text {th }}-5^{\text {th }}$ day, with instillations every 24 hours without hyperthermia. The EPIC regimens include mitomycin C, 5-Fluorouracil or taxanes $(3,4)$, cellcycle specific drugs that can be used due to the repeated applications of EPIC (unlike HIPEC). In 2001, Yu et al. published the results of a prospective randomized trial, in which 248 patients with GC without PM were randomized to receive standard surgery with or without EPIC as adjuvant treatment. The gastric resection plus EPIC group had better 5-year OS (54\% versus 38\%, P=0.0278) (47).

Most recently, in 2014, Kwon et al. reported the results of a retrospective study with 245 patients diagnosed by locally advanced (serosal invasion) GC without PM. The addition of EPIC to gastric resection improved the OS (5-year OS of $47.4 \%$ in EPIC group versus $26.7 \%$ in non-EPIC group, $\mathrm{P}=0.012$ ), mainly due to a decrease in peritoneal relapse (48).

EPIC can be added to CRS and HIPEC in patients with good performance status in experienced centers (3). However, the use of EPIC is not as widespread as that of HIPEC, because it generates more infections, more fistulas and other postoperative complications (4).

\section{Prophylactic or adjuvant HIPEC}

Peritoneal relapse is the most common pattern of recurrence after radical surgery in locally advanced GC. The use of HIPEC associated to a curative gastrectomy in patients without PM, but with a locally advanced GC, can prevent peritoneal recurrence. Several Asian studies have demonstrated that the use of HIPEC as a prophylactic treatment is effective (Table 2).

Yonemura et al. in 2001 randomized 139 patients in three groups (surgery alone, surgery plus normothermic intraperitoneal chemotherapy and surgery with HIPEC) and the survival results at 5-year were $61 \%$ in HIPEC group versus $44 \%$ and $42 \%$ in the two other groups $(\mathrm{P}=0.021)(46)$.

Different reviews and meta-analyses confirm the survival benefit of adjuvant HIPEC $(18,22,23)$. Recently, Desiderio et al. published a meta-analysis of 11 clinical trials and 21 observational studies, with more than 2,000 patients. The 5 -year OS for patients treated with adjuvant HIPEC was better than that of the patients treated with surgery alone $(\mathrm{RR}=0.82, \mathrm{P}=0.01)(23)$.

Most published studies are Asian, and perhaps for this reason the use of prophylactic HIPEC has not been extended to Western countries. Nevertheless, currently a new important clinical trial is ongoing in Europe (GASTRICHIP) and we will know if its outcomes are similar to the Asian studies when the results become available (26).

\section{Palliative treatment}

Many patients have an unresectable PM from GC, and these cases can develop malignant ascites with symptoms of abdominal pain, dyspnea, early satiety, or fatigue. 
Table 2 Published studies evaluating hyperthermic intraperitoneal chemotherapy (HIPEC) as prophylactic treatment in absence of peritoneal metastases

\begin{tabular}{|c|c|c|c|c|}
\hline Author, year & Number of patients & Drug & Morbidity & Survival \\
\hline Fujimoto 1999, (51) & $\begin{array}{c}71 \text { (surgery + HIPEC) vs. } \\
70 \text { (surgery alone) }\end{array}$ & MMC & $2 / 71$ vs. $2 / 70$ & 4-year OS: $76 \%$ vs. $58 \%$ \\
\hline Yonemura 2001, (46) & $\begin{array}{c}48 \text { (surgery + HIPEC) vs. } \\
44 \text { (surgery + NIPEC) vs. } \\
47 \text { (surgery alone) }\end{array}$ & MMC + Cisplatin & $\begin{array}{c}19 \% \text { vs. } 14 \% \text { vs. } 19 \% \\
\text { (mortality: } 4 \% \text { vs. } 0 \% \text { vs. } \\
4 \% \text { ) }\end{array}$ & $\begin{array}{c}5 \text {-year OS: } 61 \% \text { vs. } 44 \% \text { vs. } \\
42 \%\end{array}$ \\
\hline Zhu 2006, (52) & $\begin{array}{c}41 \text { (surgery + HIPEC) vs. } \\
53 \text { (surgery alone) }\end{array}$ & MMC + Cisplatin & $23 \%$ vs. $12 \%$ & 4-year OS: $70 \%$ vs. $52 \%$ \\
\hline
\end{tabular}

MMC, mitomycin C; OS, overall survival; NIPEC, normothermic intraperitoneal chemotherapy.

Laparoscopic HIPEC is an option for these patients. Some studies have reported a success rate in symptom control of $95 \%(53,54)$. Facchiano et al. published a systematic review which included 76 patients treated with palliative laparoscopic HIPEC. They reported a $95 \%$ success in ascites control, with $7 \%$ mild complications and without severe morbidity (54).

Most recently, a new technique has been described to improve the quality of life for patients with unresectable PM. Pressurized intraperitoneal aerosol chemotherapy (PIPAC) is a closed technique of chemotherapy administration. The drug is administered by laparoscopy, aerosolized by an injector at high pressure, so that it achieves a better penetration into the tissues (55). In the last two years, many studies have been published about PIPAC. All the studies are focused on palliative patients. Alyami et al. published a systematic review in 2019, which included 838 patients (185 with GC). The clinical response was $50 \%$ to $91 \%$ in patients with GC (56). Currently, there are many studies ongoing evaluating the use of PIPAC for PM, even considering its use as a neoadjuvant therapy to convert unresectable patients into patients with the chance to achieve complete cytoreduction.

The expectations of PIPAC are very high, both in the palliative setting and as neoadjuvant therapy. The results published so far are encouraging, but they are no better than those of laparoscopic HIPEC, which has been widely studied as neoadjuvant as well as palliative treatment, and it is frankly cheaper. Therefore, future studies should compare PIPAC versus laparoscopic HIPEC in order to know if PIPAC provides better results than those already demonstrated by laparoscopic HIPEC.

\section{Conclusions}

PM from GC has a poor prognosis. Patients should be managed in experienced centers in order to select those who can benefit of a multimodal treatment. Diagnostic laparoscopy is mandatory to assess the peritoneal disease and to obtain a cytology. Only patients with low volume $\mathrm{PM}$ or patients with isolated positive cytology can benefit from a multimodal and aggressive treatment, which includes an intensive neoadjuvant chemotherapy (systemic, intraperitoneal, or in combination) and CRS (including curative gastrectomy and D2 lymphadenectomy) with HIPEC. Nowadays, the HIPEC regimen is not standardized. A summary of our recommendations is shown in Table 3.

Patients with locally advanced GC (especially with serosal invasion) have a high risk of peritoneal recurrence. The use of adjuvant HIPEC in these patients has improved the OS in Asian studies, by decreasing peritoneal relapse. A French randomized clinical trial (GASTRICHIP) with Spanish collaboration is ongoing. 
Table 3 Recommendations for perioperative management for gastric cancer (GC) with peritoneal metastases (PM)

Preoperative laparoscopy is mandatory to assess $\mathrm{PCl}$ and to obtain peritoneal cytology

Patients with GC and PM should be referred to experienced centers in the treatment of peritoneal malignancies

Patients with limited and potentially resectable $\mathrm{PM}(\mathrm{PCl} \leq 6)$ should be treated with a multimodal approach

We recommend 4 preoperative cycles of systemic chemotherapy with FLOT (fluorouracil, leucovorin, oxaliplatin and docetaxel), followed by cytoreductive surgery (CRS) with HIPEC, and then another 4 cycles of FLOT

The HIPEC currently in use at Hospital of Fuenlabrada is cisplatin $75 \mathrm{mg} / \mathrm{m}^{2}$ during 60 minutes

Neoadjuvant laparoscopic HIPEC or NIPS (neoadjuvant intraperitoneal and systemic chemotherapy) have shown good results, and can be preoperatively used in experienced centers, but in the context of clinical trials

EPIC (early postoperative intraperitoneal chemotherapy) can be added to CRS and HIPEC in patients with good performance status in experienced centers

Patients with GC and unresectable PM must be treated with palliative systemic chemotherapy. Laparoscopic HIPEC or PIPAC (pressurized intraperitoneal aerosol chemotherapy) can be used to improve quality of life of the symptomatic patients, in referral centers

Patients with high volume PM can develop symptoms with marked reduction in their quality of life. Laparoscopic HIPEC or PIPAC may achieve improvement of these symptoms in a palliative setting.

The results of ongoing studies in all these clinical scenarios will provide better guidance for the treatment of these patients, probably changing the therapeutic algorithms for the management of advanced GC.

\section{Acknowledgments}

Funding: None.

\section{Footnote}

Provenance and Peer Review: This article was commissioned by the Guest Editors (Paul H. Sugarbaker and Kurt Van der Speeten) for the focused issue "Intraperitoneal Chemotherapy for Peritoneal Metastases: HIPEC, EPIC, NIPEC, PIPAC and More" published in Fournal of
Gastrointestinal Oncology. This article has undergone external peer review.

Conflicts of Interest: All authors have completed the ICMJE uniform disclosure form (available at http://dx.doi. org/10.21037/jgo-20-232). The focused issue was sponsored by the Peritoneal Surface Oncology Group International (PSOGI). The authors have no other conflicts of interest to declare.

Reporting Checklist: The authors have completed the Narrative reporting checklist. Available at http://dx.doi. org/10.21037/jgo-20-232

Etbical Statement: The authors are accountable for all aspects of the work in ensuring that questions related to the accuracy or integrity of any part of the work are appropriately investigated and resolved.

Open Access Statement: This is an Open Access article distributed in accordance with the Creative Commons Attribution-NonCommercial-NoDerivs 4.0 International License (CC BY-NC-ND 4.0), which permits the noncommercial replication and distribution of the article with the strict proviso that no changes or edits are made and the original work is properly cited (including links to both the formal publication through the relevant DOI and the license). See: https://creativecommons.org/licenses/by-nc-nd/4.0/.

\section{References}

1. Bray F, Ferlay J, Soerjomataram I, et al. Global cancer statistics 2018: GLOBOCAN estimates of incidence and mortality worldwide for 36 cancers in 185 countries. CA Cancer J Clin 2018;68:394-424.

2. Jemal A, Bray F, Center MM, et al. Global cancer statistics. CA Cancer J Clin 2011;61:69-90.

3. Gamboa AC, Winer JH. Cytoreductive surgery and hyperthermic intraperitoneal chemotherapy for gastric cancer. Cancers 2019;11:1662.

4. Macrì $\mathrm{A}$, Morabito $\mathrm{F}$. The use of intraperitoneal chemotherapy for gastric malignancies. Expert Rev Anticancer Ther 2019;19:879-88.

5. Kuramoto M, Shimada S, Ikeshima S, et al. Extensive intraoperative peritoneal lavage as a standard prophylactic strategy for peritoneal recurrence in patients with gastric carcinoma. Ann Surg 2009;250:242-6. 
6. Manzanedo I, Pereira F, Rihuete Caro C, et al. Cytoreductive Surgery and Hyperthermic Intraperitoneal Chemotherapy (HIPEC) for Gastric Cancer with Peritoneal Carcinomatosis: Multicenter Study of Spanish Group of Peritoneal Oncologic Surgery (GECOP). Ann Surg Oncol 2019;26:2615-21.

7. Bilici A. Treatment options in patients with metastatic gastric cancer: current status and future perspectives. World J Gastroenterol 2014;20:3905-15.

8. Bang YJ, Van Cutsem E, Feyereislova A, et al. Trastuzumab in combination with chemotherapy versus chemotherapy alone for treatment of HER2-positive advanced gastric or gastro-oesophageal junction cancer (ToGA): a phase 3, open-label, randomised controlled trial. Lancet 2010;376:687-97.

9. Lordick F, Kang YK, Chung H-C, et al. Capecitabine and cisplatin with or without cetuximab for patients with previously untreated advanced gastric cancer (EXPAND): a randomised, open-label phase 3 trial. Lancet Oncol 2013;14:490-9.

10. Hong SH, Shin YR, Roh SY, et al. Treatment outcomes of systemic chemotherapy for peritoneal carcinomatosis arising from gastric cancer with no measurable disease: retrospective analysis from a single center. Gastric Cancer 2013;16:290-300.

11. Rihuete Caro C, Manzanedo I, Pereira F, et al. Cytoreductive surgery combined with hyperthermic intraperitoneal chemotherapy (HIPEC) in patients with gastric cancer and peritoneal carcinomatosis. Eur J Surg Oncol 2018;44:1805-10.

12. Glehen O, Gilly FN, Arvieux C, et al. Peritoneal carcinomatosis from gastric cancer: a multi-institutional study of 159 patients treated by cytoreductive surgery combined with perioperative intraperitoneal chemotherapy. Ann Surg Oncol 2010;17:2370-7.

13. Brücher BLDM, Piso P, Verwaal V, et al. Peritoneal carcinomatosis: cytoreductive surgery and HIPEC-overview and basics. Cancer Invest 2012;30:209-24.

14. Yonemura Y, Canbay E, Li Y, et al. A comprehensive treatment for peritoneal metastases from gastric cancer with curative intent. Eur J Surg Oncol 2016;42:1123-31.

15. Sugarbaker PH. Peritonectomy procedures. Ann Surg 1995;221:29-42.

16. Sugarbaker PH. Intraperitoneal chemotherapy and cytoreductive surgery for the prevention and treatment of peritoneal carcinomatosis and sarcomatosis. Semin Surg Oncol 1998;14:254-61.

17. Sugarbaker PH. It's what the surgeon doesn't see that kills the patient. J Nippon Med Sch 2000;67:5-8.

18. Yan TD, Welch L, Black D, et al. A systematic review on the efficacy of cytoreductive surgery combined with perioperative intraperitoneal chemotherapy for diffuse malignancy peritoneal mesothelioma. Ann Oncol 2007;18:827-34.

19. Verwaal VJ, Bruin S, Boot H, et al. 8-year follow-up of randomized trial: cytoreduction and hyperthermic intraperitoneal chemotherapy versus systemic chemotherapy in patients with peritoneal carcinomatosis of colorectal cancer. Ann Surg Oncol 2008;15:2426-32.

20. Sugarbaker PH. New standard of care for appendiceal epithelial neoplasms and pseudomyxoma peritonei syndrome? Lancet Oncol 2006;7:69-76.

21. van Driel WJ, Koole SN, Sikorska K, et al. Hyperthermic Intraperitoneal Chemotherapy in Ovarian Cancer. N Engl J Med 2018;378:230-40.

22. Coccolini F, Cotte E, Glehen O, et al. Intraperitoneal chemotherapy in advanced gastric cancer. Meta-analysis of randomized trials. Eur J Surg Oncol 2014;40:12-26.

23. Desiderio J, Chao J, Melstrom L, et al. The 30-year experience-A meta-analysis of randomised and high-quality non-randomised studies of hyperthermic intraperitoneal chemotherapy in the treatment of gastric cancer. Eur J Cancer 2017;79:1-14.

24. Chia CS, You B, Decullier E, et al. Patients with Peritoneal Carcinomatosis from Gastric Cancer Treated with Cytoreductive Surgery and Hyperthermic Intraperitoneal Chemotherapy: Is Cure a Possibility? Ann Surg Oncol 2016;23:1971-9.

25. Yang XJ, Huang CQ, Suo T, et al. Cytoreductive surgery and hyperthermic intraperitoneal chemotherapy improves survival of patients with peritoneal carcinomatosis from gastric cancer: final results of a phase III randomized clinical trial. Ann Surg Oncol 2011;18:1575-81.

26. Glehen O, Passot G, Villeneuve L, et al. GASTRICHIP:

D2 resection and hyperthermic intraperitoneal chemotherapy in locally advanced gastric carcinoma: a randomized and multicenter phase III study. BMC Cancer 2014;14:183.

27. Amin MB, Greene FL, Edge SB, et al. The Eighth Edition AJCC Cancer Staging Manual: Continuing to build a bridge from a population-based to a more «personalized» approach to cancer staging. CA Cancer J Clin 2017;67:93-9.

28. Portilla AG, Shigeki K, Dario B, et al. The intraoperative staging systems in the management of peritoneal surface malignancy. J Surg Oncol 2008;98:228-31.

29. Bonnot PE, Piessen G, Kepenekian V, et al. Cytoreductive 
Surgery With or Without Hyperthermic Intraperitoneal Chemotherapy for Gastric Cancer With Peritoneal Metastases (CYTO-CHIP study): A Propensity Score Analysis. J Clin Oncol 2019;37:2028-40.

30. González-Moreno S, Kusamura S, Baratti D, et al. Postoperative residual disease evaluation in the locoregional treatment of peritoneal surface malignancy. J Surg Oncol 2008;98:237-41.

31. Rau B, Brandl A, Piso P, et al. Peritoneal metastasis in gastric cancer: results from the German database. Gastric Cancer 2020;23:11-22.

32. Cunningham D, Allum WH, Stenning SP, et al. Perioperative chemotherapy versus surgery alone for resectable gastroesophageal cancer. $\mathrm{N}$ Engl J Med 2006;355:11-20.

33. Al-Batran SE, Homann N, Pauligk C, et al. Perioperative chemotherapy with fluorouracil plus leucovorin, oxaliplatin, and docetaxel versus fluorouracil or capecitabine plus cisplatin and epirubicin for locally advanced, resectable gastric or gastro-oesophageal junction adenocarcinoma (FLOT4): a randomised, phase 2/3 trial. Lancet 2019;393:1948-57.

34. Yonemura Y, Ishibashi H, Hirano M, et al. Effects of Neoadjuvant Laparoscopic Hyperthermic Intraperitoneal Chemotherapy and Neoadjuvant Intraperitoneal/Systemic Chemotherapy on Peritoneal Metastases from Gastric Cancer. Ann Surg Oncol 2017;24:478-85.

35. Badgwell B, Blum M, Das P, et al. Phase II Trial of Laparoscopic Hyperthermic Intraperitoneal Chemoperfusion for Peritoneal Carcinomatosis or Positive Peritoneal Cytology in Patients with Gastric Adenocarcinoma. Ann Surg Oncol 2017;24:3338-44.

36. Yonemura Y, Bandou E, Sawa T, et al. Neoadjuvant treatment of gastric cancer with peritoneal dissemination. Eur J Surg Oncol 2006;32:661-5.

37. Yonemura Y, Elnemr A, Endou Y, et al. Effects of neoadjuvant intraperitoneal/systemic chemotherapy (bidirectional chemotherapy) for the treatment of patients with peritoneal metastasis from gastric cancer. Int J Surg Oncol 2012;2012:148420.

38. Canbay E, Mizumoto A, Ichinose M, et al. Outcome data of patients with peritoneal carcinomatosis from gastric origin treated by a strategy of bidirectional chemotherapy prior to cytoreductive surgery and hyperthermic intraperitoneal chemotherapy in a single specialized center in Japan. Ann Surg Oncol 2014;21:1147-52.

39. Fujiwara Y, Takiguchi S, Nakajima K, et al. Neoadjuvant intraperitoneal and systemic chemotherapy for gastric cancer patients with peritoneal dissemination. Ann Surg Oncol 2011;18:3726-31.

40. Hao Y, Liu Y, Ishibashi H, et al. Downstaging of lymph node metastasis after neoadjuvant intraperitoneal and systemic chemotherapy in gastric carcinoma with peritoneal metastasis. Eur J Surg Oncol 2019;45:1493-7.

41. González-Moreno S, González-Bayón LA, Ortega-Pérez G. Hyperthermic intraperitoneal chemotherapy: Rationale and technique. World J Gastrointest Oncol 2010;2:68-75.

42. Yonemura Y, Fujimura T, Nishimura G, et al. Effects of intraoperative chemohyperthermia in patients with gastric cancer with peritoneal dissemination. Surgery 1996;119:437-44.

43. Fujimoto S, Takahashi M, Mutou T, et al. Improved mortality rate of gastric carcinoma patients with peritoneal carcinomatosis treated with intraperitoneal hyperthermic chemoperfusion combined with surgery. Cancer 1997;79:884-91.

44. Dindo D, Demartines N, Clavien PA. Classification of surgical complications: a new proposal with evaluation in a cohort of 6336 patients and results of a survey. Ann Surg 2004;240:205-13.

45. Yonemura Y, Kawamura T, Bandou E, et al. Treatment of peritoneal dissemination from gastric cancer by peritonectomy and chemohyperthermic peritoneal perfusion. Br J Surg 2005;92:370-5.

46. Yonemura Y, de Aretxabala X, Fujimura T, et al. Intraoperative chemohyperthermic peritoneal perfusion as an adjuvant to gastric cancer: final results of a randomized controlled study. Hepatogastroenterology 2001;48:1776-82.

47. Yu W, Whang I, Chung HY, et al. Indications for early postoperative intraperitoneal chemotherapy of advanced gastric cancer: results of a prospective randomized trial. World J Surg 2001;25:985-90.

48. Kwon OK, Chung HY, Yu W. Early postoperative intraperitoneal chemotherapy for macroscopically serosainvading gastric cancer patients. Cancer Res Treat 2014;46:270-9.

49. Koga S, Hamazoe R, Maeta M, et al. Prophylactic therapy for peritoneal recurrence of gastric cancer by continuous hyperthermic peritoneal perfusion with mitomycin C. Cancer 1988;61:232-7.

50. Ikeguchi M, Kondou A, Oka A, et al. Effects of continuous hyperthermic peritoneal perfusion on prognosis of gastric cancer with serosal invasion. Eur J Surg 1995;161:581-6.

51. Fujimoto S, Takahashi M, Mutou T, et al. Successful intraperitoneal hyperthermic chemoperfusion for the prevention of postoperative peritoneal recurrence 
in patients with advanced gastric carcinoma. Cancer 1999;85:529-34.

52. Zhu ZG, Tang R, Yan M, et al. Efficacy and safety of intraoperative peritoneal hyperthermic chemotherapy for advanced gastric cancer patients with serosal invasion. A long-term follow-up study. Dig Surg 2006;23:93-102.

53. Valle M, Van der Speeten K, Garofalo A. Laparoscopic hyperthermic intraperitoneal peroperative chemotherapy (HIPEC) in the management of refractory malignant ascites: A multi-institutional retrospective analysis in 52 patients. J Surg Oncol 2009;100:331-4.

54. Facchiano E, Risio D, Kianmanesh R, et al. Laparoscopic

Cite this article as: Manzanedo I, Pereira F, Serrano Á, Pérez-Viejo E. Review of management and treatment of peritoneal metastases from gastric cancer origin. J Gastrointest Oncol 2021;12(Suppl 1):S20-S29. doi: 10.21037/jgo-20-232 hyperthermic intraperitoneal chemotherapy: indications, aims, and results: a systematic review of the literature. Ann Surg Oncol 2012;19:2946-50.

55. Esquis P, Consolo D, Magnin G, et al. High intraabdominal pressure enhances the penetration and antitumor effect of intraperitoneal cisplatin on experimental peritoneal carcinomatosis. Ann Surg 2006;244:106-12.

56. Alyami M, Hübner M, Grass F, et al. Pressurised intraperitoneal aerosol chemotherapy: rationale, evidence, and potential indications. Lancet Oncol 2019;20:e368-77. 